\title{
Reflektionen zum Stand der gestaltungsorientierten Wirtschaftsinformatik
}

\section{DOI 10.1007/s11576-014-0410-x}

\section{Der Autor}

Prof. Dr. Martin Bichler ( $\varangle)$

Decision Sciences \& Systems

Institut für Informatik

TU München

Boltzmannstraße 3

85748 München

Deutschland

bichler@in.tum.de

Online publiziert: 2014-02-12

This article is also available in English via http://www.springerlink.com and http://www.bise-journal.org: Bichler M (2014) Design Science: Impressions from ICIS 2013. Bus Inf Syst Eng. doi: 10.1007/s12599-014-0317-5.

(C) Springer Fachmedien Wiesbaden 2014
Die ICIS 2013 im schönen Mailand ist zum Zeitpunkt des Verfassens dieses Editorials gerade zu Ende gegangen und ich möchte die Gelegenheit nutzen, um einige Beobachtungen zu diskutieren, bevor ich kurz die Inhalte dieses Heftes einführe.

Für sehr viele Kollegen in der Wirtschaftsinformatik gehört die ICIS zu den wichtigsten jährlichen Tagungen. Auf der Konferenz im Dezember 2013 konnte ein Rekord von 1537 Teilnehmern verzeichnet werden. Für US-amerikanische Business Schools ist die ICIS daneben ein wichtiger akademischer Arbeitsmarkt und viele Doktoranden hatten Interviews mit Vertretern verschiedener Universitäten. Ebenso ist die Tagung ein wichtiger Gradmesser für Entwicklungen in der internationalen Wirtschaftsinformatik.

Die Konferenz ist thematisch und methodisch sehr breit und umfasst qualitative und quantitative Forschungsansätze, ebenso wie gestaltungsorientierte Arbeiten (DesignScience). Vor nunmehr 10 Jahren publizierten Hevner et al. ihren mittlerweile über 4.600 Mal zitierten Artikel „Design Science in Information Systems Research“. Ich möchte dieses Jubiläum zum Anlass nehmen, um die Tagung aus der Perspektive der gestaltungsorientierten Wirtschaftsinformatik zu reflektieren. Die Tagung ist so groß, dass man unmöglich in wenigen Absätzen ein komplettes Bild der ICIS zeichnen kann. Somit werde ich nachfolgend lediglich einen Eindruck über die Präsenz gestaltungsorientierter Forschung wiedergeben.

Die Tagung startete mit dem ICIS Doctoral Consortium und die Beiträge in diesem können als eine Stichprobe dafür herangezogen werden, welche Methoden junge Doktoranden heute einsetzen. Man sollte dabei erwähnen, dass die Mehrheit der 40 Studierenden im Doctoral Consortium an US-Universitäten tätig ist und die Veranstaltung damit nicht notwendigerweise ein repräsentatives Bild der Forschung in anderen Teilen der Welt darstellt. Quantitative Arbeiten stellten zweifellos den verbreitetsten Ansatz der Doktoranden dar. Beispielsweise wurden Daten von verschiedenen Websites wie Online-Jobbörsen, Finanzportalen oder Crowdfunding-Plattformen gesammelt, um danach das Nutzerverhalten mit mehr oder weniger fortgeschrittenen ökonometrischen Methoden zu analysieren. Insgesamt spielte angewandte Ökonometrie eine sehr große Rolle in den meisten Präsentationen, gestaltungsorientierte Forschung dafür kaum. Abhängig vom Schwerpunkt der analysierten Websites gab es starke Anknüpfungspunkte zu Forschung in der Finanzwirtschaft, im Marketing oder in anderen betriebswirtschaftlichen Disziplinen. Auch im Programm der Hauptkonferenz überwogen quantitative und qualitative Methoden gegenüber gestaltungsorientierten Arbeiten. Insgesamt lässt sich sagen, dass nach 10 Jahren intensiver Diskussion über gestaltungsorientierte Forschung in der Wirtschaftsinformatik die Anzahl der Artikel mit gestaltungsorientierten Elementen sowohl im Doctoral Consortium als auch in der Hauptkonferenz relativ klein war.

Das soll nicht als Kritik an den auf der Tagung vorgestellten Arbeiten verstanden werden. Viele Präsentationen im Doctoral Consortium und auf der ICIS haben interessante Beobachtungen über menschliches Verhalten in Onlineumgebungen zutage gebracht, die nicht offensichtlich, aber für Websitebetreiber durchaus bedeutend sind. Ich bin überzeugt davon, dass die Wirtschaftsinformatik Informationssysteme aus einer ökonomischen und verhaltenswissenschaftlichen Perspektive analysieren muss und dass einige der verhaltenswissenschaftlichen Fragestellungen in Zukunft sogar noch an Bedeutung in unserer zunehmend digitalen Welt gewinnen werden. Eine Wirtschaftsinformatik ohne verhaltenswissenschaftliche und ökonomische Beiträge ist aus meiner Sicht heute nicht mehr denkbar und auch nicht zeitgemäß. Wir müssen als wissenschaftliche Community belastbare Aussagen über die Auswirkung von Informationssystemen auf Individuen, Gruppen und Organisationen treffen können, und diese werden zunehmend wichtiger für die Gestaltung moderner Informationssysteme.

Ich glaube allerdings ebenso, dass es für unsere wissenschaftliche Disziplin langfristig von Schaden wäre, wenn wir gestaltungsorientierte Forschung aufgeben oder deutlich 
reduzieren. Einerseits hätte das starke Auswirkungen auf die Lehre. Wenn wir uns nicht mehr mit der Gestaltung von Informationssystemen in unserer Forschung beschäftigen, wird es deutlich geringere Anreize geben, entsprechende Methoden auch zu unterrichten, bzw. wird einfach die Expertise dazu an einigen Hochschulen abhanden kommen. Die meisten Doktoranden im Doctoral Consortium hatten keinen nennenswerten Hintergrund im Entwurf oder der Implementierung von Informationssystemen. Die Nachfrage nach Wirtschaftsinformatikern in der Praxis ist jedoch zu einem guten Teil auch damit zu erklären, dass Absolventen auch Informationssysteme entwerfen und gestalten können. Auch bei steigender Bedeutung statistischer und ökonometrischer Methoden für Praxis und die Wissenschaft sollten solche Methoden gestaltungsorientierte Inhalte in der Lehre ergänzen, aber nicht ersetzen.

Andererseits würden wir neben der Lehre auch in der Forschung einen Wettbewerbsvorteil anderen Disziplinen gegenüber verlieren. Viele Kollegen im Marketing analysieren Kundenverhalten auf Online-Marktpätzen. Ebenso sammeln viele Kollegen in der Finanzwirtschaft Daten aus Online-Finanzportalen und analysieren damit Investitionsentscheidungen. Das soll natürlich nicht heißen, dass Arbeiten mit entsprechenden Schwerpunkten an der Schnittstelle zu Marketing oder Finanzwirtschaft in Zeitschriften der Wirtschaftsinformatik nicht publiziert werden sollen. Gerade diese Schnittstellen sind für unsere Disziplin sehr fruchtbar. Jedoch ist viel erfolgreiche Forschung in der Wirtschaftsinformatik dadurch gekennzeichnet, dass Informationssysteme entworfen und dann analysiert werden. Bei der Analyse kommen dann Laborexperimente, Feldstudien, ökonometrische Auswertungen oder qualitative Methoden zum Einsatz. Das gestaltende Element ist aber ein wichtiger Aspekt, der viele Artikel in der Wirtschaftsinformatik von Beiträgen in anderen betriebswirtschaftlichen Disziplinen unterscheidet. Es gibt uns die Möglichkeit die digitale Welt von morgen mit zu entwerfen, gerade heutzutage eine äußerst reizvolle Aufgabe.

Im Vergleich zu großen Teilen der Informatik zeichnet sich gute gestaltungsorientierte Forschung in der Wirtschaftsinformatik auch dadurch aus, dass sozio-technische Systeme entworfen oder auch entwickelt werden und die Analyse dieser Systeme auf dem soliden Fundament sozial- und wirtschaftswissenschaftlicher Methoden zur Analyse menschlichen Verhaltens basiert. Diese Kombination ist eine besondere Stärke der Wirtschaftsinformatik und ein Mehrwert, den es zu bewahren gilt. Meine Frage an einen Doktoranden im ICIS Doctoral Consortium, warum er seine Arbeit an eine Zeitschrift in der Wirtschaftsinformatik und nicht im Marketing schickt, wurde damit beantwortet, dass eine der erklärenden Variablen im Regressionsmodell einen IT-Bezug aufweise. Für einzelne Publikationen und Bereiche der Wirtschaftsinformatik ist das durchaus in Ordnung. Als wissenschaftliche Disziplin müssen wir aber eine Antwort darauf geben können, wo die Beiträge dieser Disziplin sich von anderen unterscheiden. Diese Frage wird spätestens in Berufungskommissionen und bei der Neuausrichtung von Lehrstühlen gestellt. Die Editorial Statements der neuen BISEDepartments sollen die Identität der Teildisziplinen in der Wirtschaftsinformatik klarer kommunizieren und damit auch die Rolle gestaltungsorientierter Forschung in einzelnen Teildisziplinen.

Unter den zahlreichen Workshops rund um die ICIS hat die WITS (Workshop on Information Technology and Systems) einen traditionell starken Fokus auf gestaltungsorientierter Forschung. Einzelne Sessions beschäftigten sich mit Data-Mining, Social Analytics, Systems Optimization oder Empfehlungssystemen. Paulo Goes, der Hauptherausgeber von MIS Quarterly, hielt einen eingeladenen Vortrag mit dem Titel „Looking for Design Science Research in Top-Tier IS Journals. Has anyone seen it?“ Er berichtete darin, dass lediglich $5 \%$ der Einreichungen an MIS Quarterly gestaltungsorientierte Arbeiten seien und dass dieses Segment sich weniger stark entwickelt habe, als das vor 10 Jahren nach der Veröffentlichung des Artikels von Hevner et al. in MIS Quarterly erwartet wurde. Er rief die Teilnehmer zu Einreichungen auf und betonte, dass MIS Quarterly für Design-Science heute sehr offen ist, mit einigen neuen Editoren aus diesem Bereich. Mit der langen Tradition der WIRTSCHAFTSINFORMATIK in gestaltungsorientierter Forschung ist unsere Zeitschrift natürlich ein besonders geeignetes Medium für Design-Research.

Viele gestaltungsorientierte Forschungsprojekte entstehen in Zusammenarbeit mit Industriepartnern. Neben dem wissenschaftlichen Anspruch haben solche Projekte oft signifikanten Einfluss auf reale Geschäftsprozesse in Unternehmen. Diese Art von Forschungstransfer ist ein wichtiger Beitrag unserer Disziplin, sie findet aber oft nicht ausreichend akademische Anerkennung. Seit 2008 vergibt die INFORMS Information Sys- 
tems Society (ISS) jährlich den ISS Design Science Award (http://mis.eller.arizona.edu/ events/informs/winners/index.asp).

In diesem Jahr wird die Zeitschrift WIRTSCHAFTSINFORMATIK als Sponsor des ISS Design Science Award auftreten, um weitere Anreize für qualitativ hochwertige und gestaltungsorientierte Forschung zu setzen. Eine Reihe von Departments der WIRTSCHAFTSINFORMATIK publizieren im Schwerpunkt gestaltungsorientierte Arbeiten, und die europäische Wirtschaftsinformatik hat das auch bereits lange vor der Publikation des Artikels von Hevner et al. in 2004 getan. Ich möchte alle Kollegen, die gestaltungsorientierte Forschungsarbeiten mit praktischer Relevanz publiziert haben, ermuntern, diese Projekte auch für den ISS Design Science Award in diesem Jahr einzureichen.

Nachfolgend werde ich die Artikel in dieser Ausgabe der Zeitschrift kurz einführen. Die ersten drei Artikel stammen noch aus Einreichungen zum Schwerpunktheft 1/2014 „Aktuelle Forschungsfelder der Wirtschaftsinformatik“. Der Aufsatz „Soziale Wissensumgebungen“"von Bick et al. beschreibt Strategien, Management und Prozesse zur Nutzung sozialer Softwaresysteme, um Aufgaben im Wissensmanagement zu verbessern. Daraus wird eine Reihe von Forschungsfragen abgeleitet. Böhmann et al. beschreiben „Service-Systems-Engineering“ als den systematischen Entwurf und die Entwicklung von Informationssystemen im Dienstleistungssektor bzw. zur Erbringung elektronischer Dienstleistungen. Sie schlagen gestaltungsorientierte Forschung ebenso wie Aktionsforschung als methodische Ansätze zum Entwurf innovativer Servicesysteme vor. Der Artikel „Social Media Analytics“ von Stieglitz et al. diskutiert Methoden und Werkzeuge zur Auswertung von Nutzerverhalten in sozialen Medien. Dazu gehören Textmining, soziale Netzwerkanalyse und Trendanalyse, die es Wissenschaftlern und Praktikern erlauben sollen, Kundenverhalten online besser zu verstehen.

Ein State-of-the-Art-Artikel von Diebold et al. diskutiert „Kursallokation und stabile Matchingverfahren“. Der Artikel entstand aus einem Projekt mit längerer Historie, in dem stabile Matchingverfahren für Zuweisungsprobleme an Universitäten evaluiert wurden. Der Nobelpreis für Wirtschaftswissenschaften im Herbst 2012 an Lloyd Shapley und Alvin Roth für stabile Matchingverfahren hat der Thematik dann eine unerwartete Popularität verschafft. Trotz dieser Popularität gibt es wenig empirische Forschung zu dem Thema. Neben einer aktuellen Literaturübersicht berichten die Autoren Ergebnisse aus zwei Feldstudien, welche Entscheidern ein klareres Bild über die Vor- und Nachteile stabiler Matchingverfahren gegenüber einfacher Zuordnung nach dem Windhundprinzip geben, wie sie an den meisten Universitäten praktiziert wird.

Schließlich bietet die Rubrik Meinung/Dialog eine Sammlung von Berichten von sechs deutschsprachigen Wissenschaftlern, die große Teile Ihrer Karriere im den USA oder in Australien verbracht haben oder das noch immer tun. Die Artikel wurden von den Kollegen Torsten Eymann, Dennis Kundisch und Jan Recker zusammengestellt und bieten eine hoch interessante Sammlung von Erfahrungsberichten und Beobachtungen über die Unterschiede wissenschaftlicher Systeme auf den verschiedenen Kontinenten. Ich hoffe, die Lektüre macht Ihnen ebenso viel Spaß wie mir. 